\title{
Chemotherapy is effective for stage I gastric cancer in patients with synchronous esophageal cancer
}

\author{
Hiroki Osumi ${ }^{1} \cdot$ Toshiyuki Yoshio $^{1} \cdot$ Keisho Chin $^{1} \cdot$ Mariko Ogura $^{1} \cdot$ \\ Yosuke Kumekawa $^{1}$. Mitsukuni Suenaga ${ }^{1} \cdot$ Satoshi Matsusaka $^{1} \cdot$ Eiji Shinozaki $^{1}$. \\ Yuji Miyamoto $^{1} \cdot$ Kenjiro Morishige $^{1} \cdot$ Akiyoshi Ishiyama $^{1}$ - Toshiaki Hirasawa ${ }^{1}$. \\ Tomohiro Tsuchida $^{1}$ - Yorimasa Yamamoto ${ }^{1}$ Junko Fujisaki ${ }^{1} \cdot$ Masahiro Igarashi $^{1}$. \\ Nobuyuki Mizunuma ${ }^{1}$
}

Received: 10 February 2015/Accepted: 10 July 2015/Published online: 11 August 2015

(C) The International Gastric Cancer Association and The Japanese Gastric Cancer Association 2015

\begin{abstract}
Background Diagnostic endoscopy occasionally shows synchronous early gastric cancer (EGC) and esophageal cancer (EC) in the same patient. The treatment plan for these comorbid cancers is unclear because, as EGC is commonly treated surgically, information on postchemotherapy outcomes for EGC are lacking, although chemotherapy and chemoradiotherapy are important in treating EC. Here, we evaluated whether unresected EGC could be safely observed while synchronous EC is treated with chemotherapy in patients with both cancers.

Methods We enrolled 30 patients with both EGC and EC who were treated with 5-FU plus cisplatin (FP) from January 2006 to September 2013, and who were evaluated with endoscopy before chemotherapy, and approximately every 3 months afterwards.

Results The response rate to FP for EGC was $46.8 \%$. Notably, five cases $(16.7 \%)$ had clinically complete responses with no progressive disease. Progression-free survival was $100 \%$ at 6 months and $96.2 \%$ at 1 year. In univariate analysis, FP was significantly more effective for mixed-type and undifferentiated adenocarcinoma than for differentiated adenocarcinoma.

Conclusions FP was effective for EGC. EGC was stable without progression for more than 6 months while patients underwent FP treatment for EC. We consider observing EGC with no treatment during chemotherapy for EC to be appropriate disease management.
\end{abstract}

Keisho Chin

kchin@jfcr.or.jp

1 Department of Gastroenterology, Cancer Institute Hospital, Japanese Foundation for Cancer Research, 3-8-31 Ariake, Koto-ku, Tokyo 135-8550, Japan
Keywords Early gastric cancer - Chemotherapy · Esophageal cancer · 5-FU · Cisplatin

\section{Introduction}

Modern diagnostic esophagogastroduodenoscopy (EGD) allows diagnosis of gastric cancer (GC) in its early stages, which is usually treated with endoscopic submucosal dissection (ESD) or surgery for clinical intramucosal (T1a) and submucosal (T1b) early gastric cancer (EGC). Although advanced and metastatic GC are commonly treated with chemotherapy, it is less commonly used to treat EGC, and so outcomes of chemotherapy for EGC are still unknown.

Concurrent chemoradiotherapy (CRT), which consists of 5-fluorouracil (5-FU) plus cisplatin (CDDP) (FP) and radiation (FPRT), has already been demonstrated to be an effective treatment option for esophageal cancer (EC) [1, 2]. Stage I EC patients who received CRT had an $80 \%$ 4-year survival rate in the prospective trial (JCOG 9708 study) [3]; these optimistic outcomes indicate that CRT is as good a choice as endoscopic resection and surgery for patients with stage I EC, although the local recurrence rate remains high. For patients with stage II/III EC, surgery after neoadjuvant chemotherapy (NAC) is the standard treatment, with definitive CRT as a nonsurgical option. In the JCOG 9906 trial, which evaluated the efficacy and toxicity of CRT with FP for stage II/III EC, the 5-year survival rate was $36.8 \%$ [4]. On the other hand, in JCOG9907, which evaluated optimal perioperative timing before or after surgery for providing chemotherapy, preoperative chemotherapy with FP was superior and its 5-year survival rate was $60 \%$ [5]. According to these results, we regarded NAC with FP and subsequent 
esophagectomy is the standard treatment for patients with stage II/III, with CRT as a second choice. In 4.4-7.9\% of $\mathrm{EC}$, synchronous GC is detected at diagnosis by detailed endoscopic examination [6, 7]. Unsurprisingly, the prognosis of patients with another primary cancer in addition to $\mathrm{EC}$ is worse than for patients with single malignancies; and deciding whether to treat first EC or EGC in these patients can be difficult. When we perform chemotherapy or CRT for EC, synchronous EGC is usually resected after completion of EC treatment because of the worse prognosis of advanced EC. However, when we perform chemotherapy or CRT for EC and EGC, we do not know whether chemotherapy for EC is effective for EGC, or whether EGC resection should be performed beforehand. No report of response rate and progression-free survival (PFS) for EGC has been published. This study evaluated the efficacy of chemotherapy for EGC, appropriate treatment order for synchronous double cancers, and appropriate follow-up intervals of EGD for EGC.

\section{Methods}

This study has been performed in accordance with the Declaration of Helsinki. Our Institutional Review Board approved this study (Registry number: 1545).

\section{Study population}

Of 329 patients who received chemotherapy and chemoradiotherapy (FP or FPRT) for histopathologically confirmed esophageal cancers between January 2006 and September 2013 at our hospital, we finally enrolled 30 patients with histopathologically confirmed EC and synchronous EGC.

Our selection criteria were (a) patients who were diagnosed with EC and treated with chemotherapy or chemoradiotherapy from January 2006 to September 2013; (b) patients had EGC simultaneously with their EC; (c) each patient's EC was confirmed histopathologically to be squamous cell carcinoma (SCC) or adenocarcinoma and EGC were confirmed as adenocarcinoma (papillary, tubular, poorly differentiated, signet-ring, mucinous, etc.); (d) EGC could be evaluated endoscopically before and after chemotherapy or chemoradiotherapy, including goodquality endoscopic images.

We excluded patients who (a) had EGCs in their irradiation area and/or (b) had prior history of surgery for EC.

Figure 1 shows the CONSORT diagram for patient selection in this study.

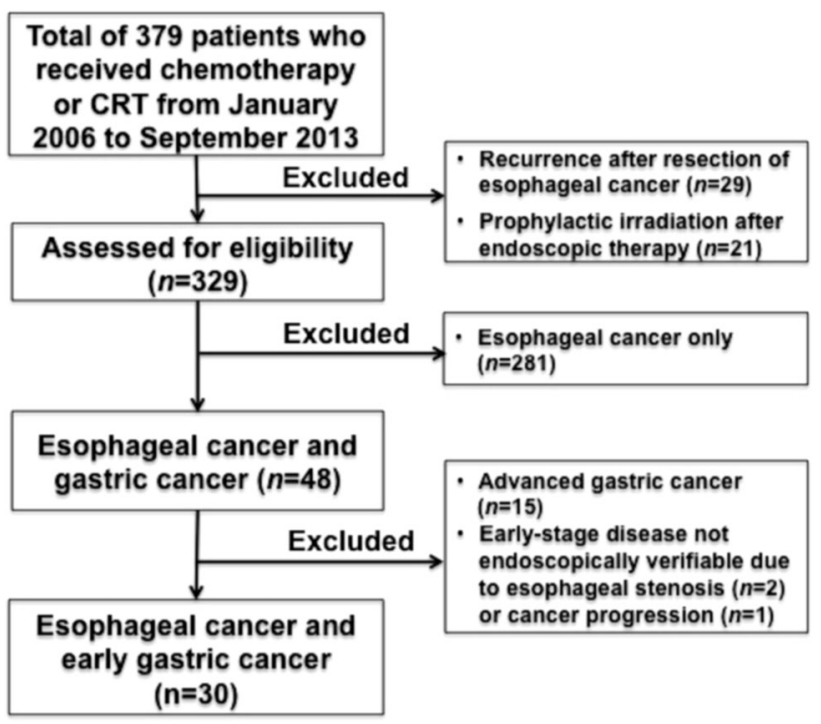

Fig. 1 CONSORT diagram of patient selection for this study

\section{Treatment}

The chemotherapy for these patients was the FP regimen, which consisted of intravenous cisplatin $\left(80 \mathrm{mg} / \mathrm{m}^{2}\right)$ on day 1 , and a continuous infusion of 5 -fluorouracil $(800 \mathrm{mg} /$ $\mathrm{m}^{2} /$ day) for days $1-5$, given every 4 weeks for two courses. Radiation therapy of 50-60 Gy for 25-30 fractions over 5-6 weeks was combined concurrently with two courses of FP therapy (CDDP $70 \mathrm{mg} / \mathrm{m}^{2}$ day 1, $29+5$-FU $700 \mathrm{mg} /$ $\mathrm{m}^{2} /$ days day $1-4$, day 29-32). None of the gastric cancers included the irradiation area.

\section{Treatment evaluation and schedule}

Patients received CT and endoscopy as treatment evaluations approximately every 3 months for those who were receiving definitive and palliative chemotherapy or CRT, and every month for those who received neoadjuvant chemotherapy (NAC) after completion of chemotherapy or CRT. EC responses to treatment were assessed through review of medical records and/or endoscopic images; and EGC responses to treatment assessed by endoscopic images (as we usually store 40-80 images for every EGD). The endoscopic images were evaluated by three certified endoscopists, and a consensus of their opinions was adopted for each patient, or after discussion among the three endoscopists in cases of wide disagreement.

The treatment response of each EGC patient was categorized as complete response (CR), partial response (PR), stable disease (SD), or progressive disease (PD). CR was 
defined that disappearance of all tumor lesions and no diagnosis of cancer in biopsy specimens. If no biopsy had been taken from the scar, we defined it as a clinical CR, which is included in PR. PR was defined as noticeable regression and flattening of a tumor on endoscopic examinations, which roughly corresponds to $\geq 50 \%$ decrease in tumor size. SD was defined as less change in tumor size or shape than for PR, but not PD. PD was defined as increased tumor size and/or worsened shape (lesions increased by $\geq 20 \%$ ). Concordance rates for CR, clinical CR, and PR were $100 \%, 100 \%$, and $27.2 \%$ among the three endoscopists, respectively. All categories were defined in accordance with Japanese Classification of Gastric Carcinoma, third English edition [8].

\section{Statistical analysis}

Overall survival (OS) and PFS were estimated using the Kaplan-Meier method and compared using the log-rank test. OS was defined as the time from beginning of CRT until death. PFS was defined as the time from beginning of CRT until objective tumor progression or death, whichever occurred first. All statistical analyses were performed with EZR (Saitama Medical Center, Jichi Medical University), which is a graphical user interface for $\mathrm{R}$ (The $\mathrm{R}$ Foundation for Statistical Computing). All $P$ values were two sided; $P<0.05$ was considered significant.

\section{Results}

\section{Characteristics of patients and lesions}

We found 32 EGC lesions in 30 patients, who had synchronous EC and were treated by FP. Median age at diagnosis was 68 years (range, 48-78 years). Median follow-up duration was 23 months. Of the 30 patients, 5 $(16.6 \%)$ were treated as NAC, $22(73.3 \%)$ as definitive FPRT and $3(10 \%)$ as palliative FPRT, for the treatment of synchronous EC. Patients underwent a median of 2.8 courses of FP (Table 1).

The 32 EGC lesions included 19 T1a cancers and 13 T1b cancers in our endoscopic diagnosis, including EUS; median tumor size was $15 \mathrm{~mm}$ (range, 6-80 mm). The patients received a median of four EGDs over median intervals of 3.1 months (Table 2).

\section{Clinical outcomes of chemotherapy for EGC}

The response rate to FP for EGC was $46.8 \%$ (CR in 2 cases and PR in 13 cases; Table 3). Notably, 2 cases achieved $\mathrm{CR}$ and 3 more cases achieved clinical CR, for a total of 5 cases $(17 \%)$ whose EGC disappeared through
Table 1 Characteristics of esophageal cancer in 30 patients

\begin{tabular}{ll}
\hline Sex (male/female) & $28 / 2$ \\
Age, median (years) & $68(48-78)$ \\
Tumor location of EC (upper/middle/lower) & $11 / 15 / 4$ \\
Histology (SCCE/ACE) & $29 / 1$ \\
PS (0/1) & $25 / 5$ \\
T stage (1/2/3/4a/4b) & $7 / 5 / 9 / 7 / 2$ \\
N stage (0/1/2/3) & $9 / 20 / 1 / 0$ \\
M stage (0/1) & $23 / 7$ \\
UICC-TNM stage, number (I/II/II/IV) & $7 / 3 / 13 / 7$ \\
Purpose of treatment (NAC/definitive/palliative) & $5 / 22 / 3$ \\
Chemotherapy (FP/FPRT) & $8 / 22$ \\
Number of chemotherapy courses (median, range) & $2.8(1-9)$ \\
Response of chemotherapy for EC (CR/PR/SD/PD) & $14 / 1 / 9 / 6$ \\
Median follow-up period, months (range) & $23(3-151)$ \\
\hline
\end{tabular}

$E C$ esophageal cancer, SCCE squamous cell carcinoma of the esophagus, $A C E$ adenocarcinoma of the esophagus, NAC neoadjuvant chemotherapy, FP fluorouracil + platinum, FPRT FP + radiation therapy, $C R$ complete response, $P R$ partial response, $S D$ stable disease, $P D$ progressive disease

Table 2 Characteristics of 32 early gastric cancer lesions in 30 patients, before chemoradiation therapy

\begin{tabular}{ll}
\hline Tumor size, median, mm (range) & $15(6-80)$ \\
Tumor location (upper/middle/lower) & $7 / 12 / 13$ \\
Macroscopic type (protruded/elevated/depressed) & $5 / 1 / 26$ \\
Pathology (differentiated/undifferentiated/mixed) & $24 / 5 / 3$ \\
TNM stage, number (Ia/Ib) & $19 / 13$ \\
Median number of EGDs undergone (range) & $4(2-12)$ \\
Median interval between EGD, months (range) & $3.1(1.1-14.8)$ \\
Median total observation period, months (range) & $4.9(1.8-49.9)$ \\
\hline
\end{tabular}

EGD esophagogastroduodenoscopy

Table 3 Response rate of early gastric cancer to a $5-\mathrm{FU}+\mathrm{CDDP}$ regimen

\begin{tabular}{lc}
\hline Response & $n(\%)$ \\
\hline $\mathrm{CR}$ & $2(6.2)$ \\
$\mathrm{PR}$ & $13(40.6)^{\mathrm{a}}$ \\
$\mathrm{SD}$ & $17(53.1)$ \\
$\mathrm{PD}$ & $0(0)$ \\
\hline$C R$ complete response, $P R$ par- \\
tial response, $S D$ stable disease, \\
$P D$ progressive disease \\
a Three patients had clinical \\
CRs
\end{tabular}

chemotherapy (Table 3), none of whom later had PD after their chemotherapy during a median observation period of 6.2 months. Of the $2 \mathrm{CR}$ cases, 1 continued to be $\mathrm{CR}$ for 47.8 months and the other case for 4 months until the 
present. The remaining 3 cases died of EC without recurrence of GC. The median PFS for all 30 patients was 32.3 months; their PFS was $100 \%$ at 6 months and $96.2 \%$ at 1 year; their overall survival rate (OS) was $23 \%$ at 3 years, and $10 \%$ at 5 years (Fig. 2). No patient died of EGC, and we confirmed PD only in 3 cases over a median observation period of 20.5 months. We performed a curative ESD for 1 patient. Two patients were observed without resection, as their EC was too advanced. Figure 3 shows the patients' clinical outcomes.
Univariate analysis for chemotherapy efficacy (Table 4)

We compared efficacy of FP by age ( $<65$ or $\geq 65$ years old), sex, EGC location (upper, middle, or lower), response of EC (CR and PR or SD and PD), lesion size $(<20$ or $\geq 20 \mathrm{~mm}$ ), macroscopic type (protruded, elevated, or depressed), pathology (differentiated, undifferentiated, or mixed), and TNM classification for GC (T1a or T1b). Of these factors, only pathology offered significant
Fig. 2 Overall and progressionfree survival for 30 patients with esophageal cancer (EC) and synchronous early gastric cancer (EGC), in whom the EC was treated by FP or FPRT. These results indicate that leaving EGC unresected during treatment for EC is an appropriate management choice
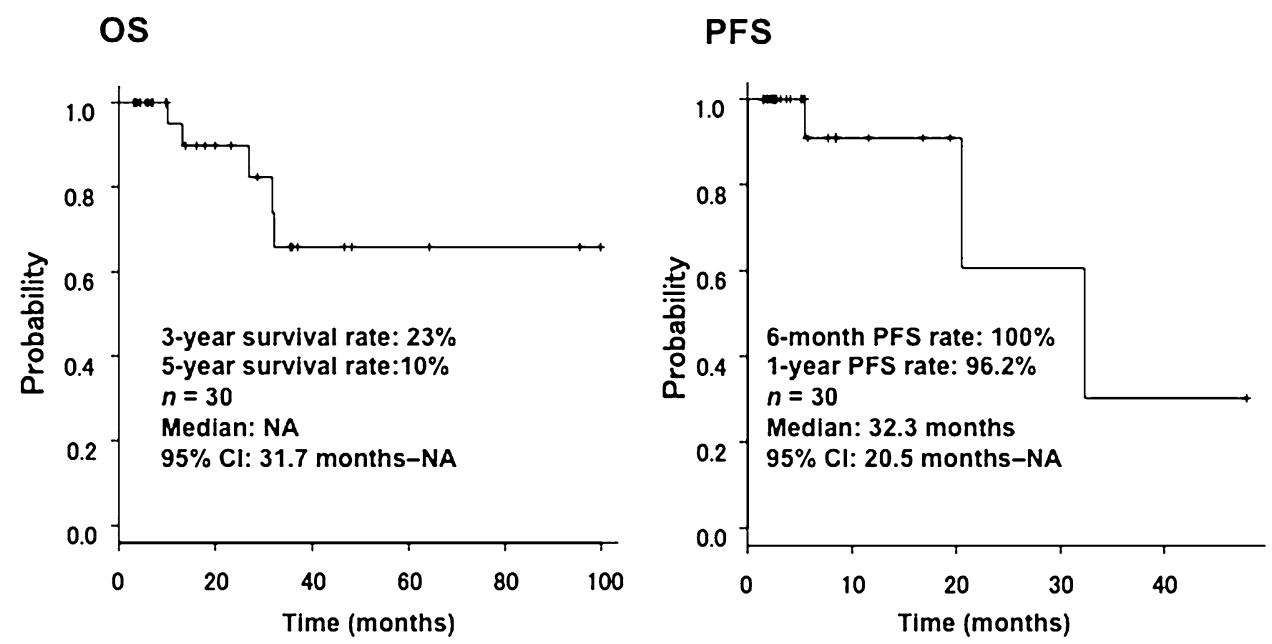

Cl: confidence interval; NA: not available; OS: overall survival; PFS: progression-free survival.

Fig. 3 Outcomes and clinical courses of patients according to treatment response of EGC after treatment of FP or FPRT

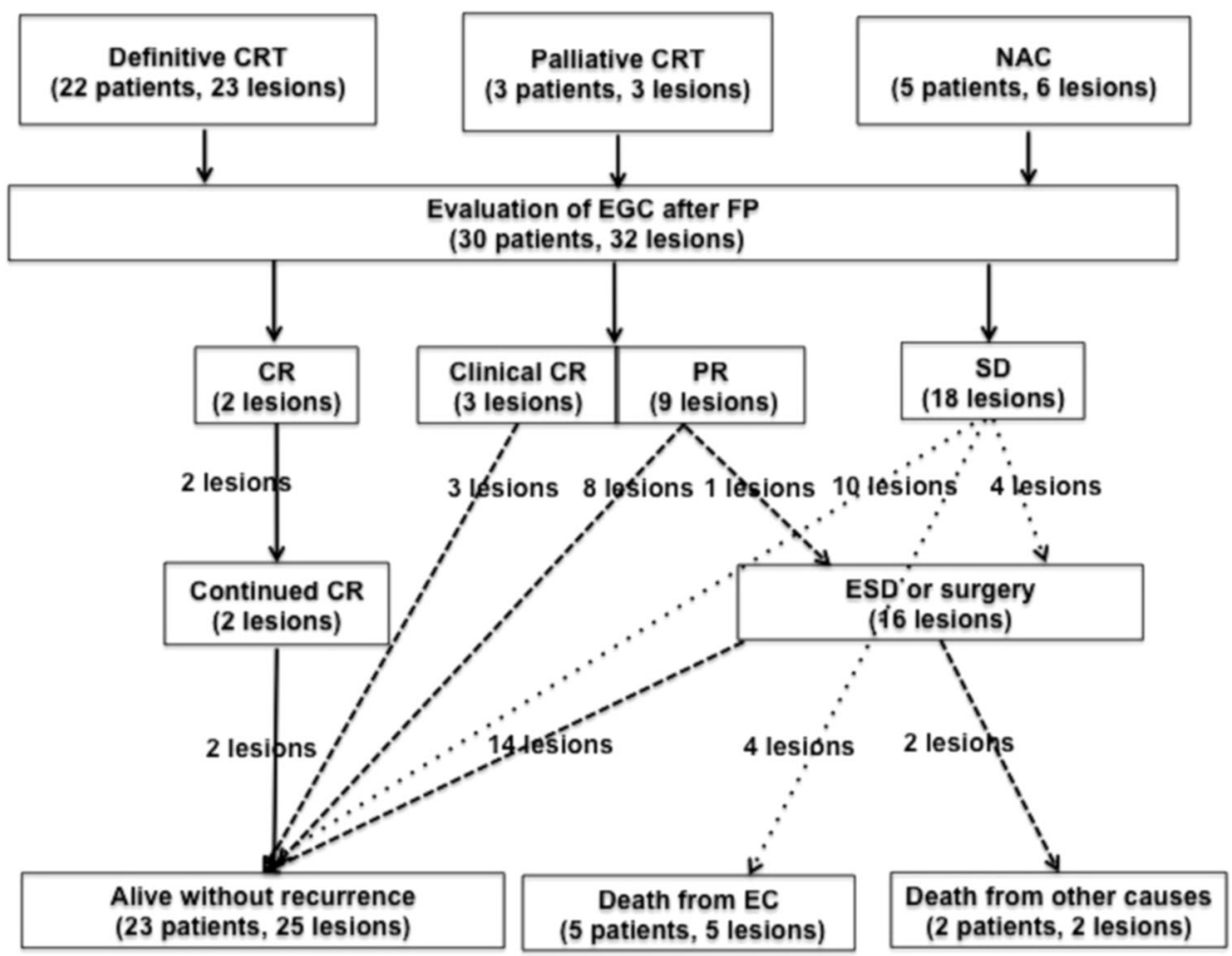


Table 4 Explorative analysis of early gastric cancer $(n=30)$

\begin{tabular}{llll}
\hline & CR, PR $(n)$ & SD $(n)$ & $P$ \\
\hline Sex (male/female) & $11 / 2$ & $17 / 0$ & 0.18 \\
Age $(<65, \geq 65)$ & $5 / 8$ & $5 / 12$ & 0.71 \\
Tumor size $(<20, \geq 20)$ & $6 / 7$ & $9 / 8$ & 1 \\
Tumor location (upper/middle/lower) & $5 / 4 / 4$ & $2 / 8 / 7$ & 0.29 \\
Macroscopic type (protruded/elevated/depressed) & $1 / 3 / 9$ & $0 / 4 / 13$ & 0.81 \\
Pathology (differentiated/undifferentiated/mixed) & $7 / 2 / 4$ & $16 / 1 / 0$ & 0.007 \\
TNM stage, number (Ia/Ib) & $6 / 7$ & $11 / 6$ & 0.46 \\
Response of chemotherapy for EC (CR, PR/SD, PD) & $12 / 1$ & $11 / 6$ & 0.1 \\
\hline
\end{tabular}

differences: FP was significantly more effective for mixedtype and undifferentiated adenocarcinoma than for differentiated adenocarcinoma.

\section{Discussion}

To our knowledge, this study is the first to evaluate the efficacy of chemotherapy for EGC. In this study, we found that the response rate of FP for EGC was $46.8 \%$ and that 2 cases achieved CR (6.2\%) and 3 more cases achieved clinical CR (9.3\%). We also showed a median PFS for EGC with synchronous EC to be 32.3 months. Under FP therapy, PFS rates at 6 months and 1 year were $100 \%$ and $96.2 \%$, respectively. Thus, when patients undergo chemotherapy or CRT for EC before the resection of EGC, they have at least 6 months, which is enough time for CRT in stage I EC, or for NAC ( 2 courses of FP) followed by esophagectomy in stage II-III EC.

Of 32 cases, 2 patients achieved CR and 3 more patients achieved clinical $\mathrm{CR}$, which is rarely reported, including 2 patients who continue to demonstrate $\mathrm{CR}$ even now. The other 3 patients died of EC without progressive GC. Of 3 patients with progressive $\mathrm{GC}$, we performed a curative ESD resection for 1 patient, but not for the other 2 patients, whose EC was too advanced. Appropriate scheduling for endoscopies is thus important so that the optimal timing of resections for EGC will not be missed.

In the mechanism of FP therapy, CDDP inhibits methionine uptake into tumor cells, causing depletion of folate cofactors including 5-CH3FH4 and $\mathrm{FH} 4$, which are essential for forming a ternary complex with thymidylate synthetase and 5-fluorodeoxyuridine, an active metabolite of 5-FU [9]. A model of GC in nude mice showed combined 5-FU and CDDP chemotherapy is useful for advanced or recurrent GC [10], because CDDP both potentiates and modulates 5-FU chemotherapy [11]. Furthermore, FP demonstrated a higher response rate $(32.5 \%$ vs. $11 \%$ ) and longer PFS than did FU alone (3.9 vs. 1.9 months), although no differences in overall survival were observed between the arms (JCOG9205) [12]. The response rate to FP for EGC was $46 \%$ in this study, which was better than the response in JOCG 9205, in which FP for advanced $\mathrm{GC}$ was addressed [12]. We think one reason for this difference was that EGC lesions were smaller, with less heterogeneous cells. Reportedly, mucosal blood flow in GC is higher than in intact mucosa and higher in early cancers than in advanced-stage disease [13].

For stage IV GC, FP is an older regimen, used less now as new drugs achieve higher response rates and longer OS, such as XPT (xeloda, cisplatin, trastuzumab), SP (TS-1 and cisplatin), or taxanes. We believe these new drugs can achieve higher response rates than FP, and possibly higher response rates for EGC than for stage IV GC. Similarly, one case report showed FOLFOX to be effective for EGC accompanying colon cancer [14], but it was only one case report; no study on the efficacy of chemotherapy for EGC has apparently been published.

In evaluating the treatment effect for EGC, ascertaining whether the progression-free-interval is a benefit from FP is difficult, because EGC also has a rather stable natural course. Tsukuma et al. reported on the natural course of EGC and showed the cumulative 5-year risk for progressing to the advanced stage was $63.0 \%$ [15]. However, our result showed a $46.8 \%$ response rate, including two cases of CR and three cases of clinical CR, which would not occur in the natural course of the disease, nor would longer PFS intervals. Although the stable nature of EGC is a consideration, our treated subjects showed a median PFS for EGC of more than 6 months.

Reportedly, the 5-year survival rates of stage I and stage II/III EC were about $80 \%$ and $60 \%$, respectively [3, 5]. In this study, $83 \%$ of the enrolled cases were stage I-III EC. So, we should consider curative treatment for both EC and EGC. Simultaneous surgery for both diseases is one possible choice. However, especially in stage II/III EC, NAC before surgery has been proven to be beneficial for OS, according to the results of JCOG9907 trial [5]. Thus, if we plan surgery for both EC and EGC, we think the ideal treatment strategy is to initially provide NAC for EC and then perform surgery for both. Our results indicate that in such cases, we need not worry too much that EGC will 
progress during NAC and can concentrate on treating the EC. On the other hand, most clinical trials exclude synchronous active cancer cases, and it has been difficult to indicate the appropriate strategy for these EGC with EC cases from prospective studies. This report is the largest number report at present and will provide instruction for daily clinical practice. Therefore, despite the relatively few cases in this study, we believe that this is important additional information for such a rarely investigated subject.

The present study has some limitations. It was a retrospective study with a small sample size. As the median follow-up period for GC was shortened by patients undergoing ESD or surgery after EC treatment, we do not know how long CR and PFS are likely to continue.

This study found that the median PFS of FP for EGC was 32.3 months after chemotherapy, and EGC did not progress for at least 6 months in any of these patients. Median intervals between EGD were 3.1 months, and we could perform curative resection for all cases, even if EGC recurred or progressed. Therefore, when we perform EGD after chemotherapy or CRT to evaluate EC, we recommend evaluating both EC and EGC every 3 months.

\section{Conclusions}

It is suggested that FP was effective for EGC. In all cases, EGC was stable without progression for 6 months during FP treatment for EC. We consider that observing EGC without resection during chemotherapy for $\mathrm{EC}$ is an appropriate choice in managing this challenging comorbidity. A further prospective study is needed to confirm the treatment strategy for EGC with EC.

Acknowledgments This investigation received no outside financial support.

\section{References}

1. Herskovic A, Martz K, al-Sarraf M, Leichman L, Brindle J, Vaitkevicius V, et al. Combined chemotherapy and radiotherapy compared with radiotherapy alone in patients with cancer of the esophagus. N Engl J Med. 1992;326:1593-8.
2. Ohtsu A, Boku N, Muro K, Chin K, Muto M, Yoshida S, et al. Definitive chemoradiotherapy for T4 and/or M1 lymph node squamous cell carcinoma of the esophagus. J Clin Oncol. 1999;17:2915-21.

3. Kato H, Sato A, Fukuda H, et al. A phase II trial of chemoradiotherapy for stage I esophageal squamous cell carcinoma: Japan clinical oncology group study (JCOG9708). Jpn J Clin Oncol. 2009;39:638-43.

4. Kato K, Muro K, Minashi K, et al. Phase II study of chemoradiotherapy with 5-fluorouracil and cisplatin for stage II-III esophageal squamous cell carcinoma: JCOG trial (JCOG 9906). Int J Radiat Oncol Biol Phys. 2011;81(3):684-90.

5. Ando N, Kato H, Igaki $\mathrm{H}$, et al. A randomized trial comparing postoperative adjuvant chemotherapy with cisplatin and 5-fluorouracil versus preoperative chemotherapy for localized advanced squamous cell carcinoma of the thoracic esophagus (JCOG9907). Ann Surg Oncol. 2012;19(1):68-74.

6. Kagei K, Hosokawa M, Shirato H, et al. Efficacy of intense screening and treatment for synchronous second primary cancers in patients with esophageal cancer. Jpn J Clin Oncol. 2002;32: $120-7$.

7. Matono S, Sueyoshi S, Tanaka T, et al. Clinical analysis of esophageal cancer associated with other primary malignancies. Jpn J Gastroenterol Surg. 2004;37:633-9.

8. Sano T, Kodera Y, Japanese Gastric Cancer Association. Japanese classification of gastric carcinoma: 3rd English edition. Gastric Cancer. 2011;14:101-12.

9. Kubota T. Theoretical basis for low-dose CDDP/5-FU therapy. Jpn J Cancer Chemother. 1999;26(11):1536-41.

10. Yamada T, Tanase M, Watanabe H, et al. Evaluation of combination chemotherapy with 5-FU and CDDP for human gastric carcinoma transplanted into nude mice. Jpn J Cancer Cemother. 1997;24(1):43-7.

11. Hirata K, Yamamitsu S, Tsuji A, et al. Biochemical modulation of 5-FU: effect of low dose CDDP. Gan To Kagaku Ryoho. 1999;26(4):467-75 (in Japanese).

12. Ohtsu A, Shimada Y, Shirao K, et al. Randomized phase III trial of fluorouracil alone versus fluorouracil plus cisplatin versus uracil and tegafur plus mitomycin in patients with unresectable, advanced gastric cancer: the Japan Clinical Oncology Group Study (JCOG9205). J Clin Oncol. 2003;21:54-9.

13. Okuhira M. Gastric mucosal blood flow early gastric cancer. Gastroenterol Endosc 1987;29(9).

14. Shimoishi K, Muto Y, Asakage M, et al. The chemotherapy with mFOLFOX6 for advanced colon cancer was effective for gastric cancer-a case report. Gan To Kagaku Ryoho (in Japanese). 2012;39:1127-30.

15. Tsukuma H, Oshima A, Narahara H, et al. Natural history of early gastric cancer: a non-concurrent, long term, follow-up study. Gut. 2000;47(5):618-21. 